\title{
A Students Attendance System Using QR Code
}

\author{
Fadi Masalha \\ Faculty of Information Technology \\ Applied Science University
}

\author{
Nael Hirzallah \\ Faculty of Information Technology \\ Applied Science University
}

\begin{abstract}
Smartphones are becoming more preferred companions to users than desktops or notebooks. Knowing that smartphones are most popular with users at the age around 26, using smartphones to speed up the process of taking attendance by university instructors would save lecturing time and hence enhance the educational process. This paper proposes a system that is based on a QR code, which is being displayed for students during or at the beginning of each lecture. The students will need to scan the code in order to confirm their attendance. The paper explains the high level implementation details of the proposed system. It also discusses how the system verifies student identity to eliminate false registrations.
\end{abstract}

Keywords-Mobile Computing; Attendance System; Educational System; GPS

\section{INTRODUCTION}

Taking students' attendance by university instructors during each class is a time consuming process especially when classes are big. Some faculty policies require this task to be performed by the instructor in each lecture. In other words, out of the total hours that are assigned to a given course, which is typically forty-five hours per semester, up to eight hours may be lost to perform this process that usually takes around ten minutes per lecture.

Statistics in [1] shows that $42 \%$ of smartphone users have an average age of 26 years old. Thus, with the widespread of smartphones among university students, this paper addresses the problem of such a waste in the lecture time and proposes a system that offers to reduce it by almost $90 \%$. The proposed solution offers a QR code for the students to scan it via a specific smartphone application. The code along with the student identity taken by the application will confirm the students' attendance.

This way, the system will save not only time but also efforts that were supposed to be put by instructors during each lecture. It will speed up the process of taking attendance and leave much time for the lecture to be given properly.

The proposed system also takes care of preventing unauthorized attendance registration using multi-factor authentication. That is, it considers "Something you know", "Something you have", and "Something you are" to confirm the student identity.

In what follows, we will discuss some related work in section 2. In section 3, we will give an overview to QR codes. In section 4, we will explain how the system works, and finally in section 5, we will conclude the paper.

\section{RELATED APPROACHES/WORK}

There are many proposals for Automatic Attendance Systems in the literature and in the market. Most of them do focus on applications to be installed on the lecturer device, whether a smartphone or a laptop. In the section, we will mention briefly few of these proposals.

Reference [2] proposes software to be installed in the instructor's mobile telephone. It enables it to query students' mobile telephone via Bluetooth connection and, through transfer of students' mobile telephones' Media Access Control (MAC) addresses to the instructor's mobile telephone; presence of the student can be confirmed.

Reference [3] is another example on a proposal that uses real time face detection algorithms integrated on an existing Learning Management System (LMS). It automatically detects and registers students attending on a lecture. The system represents a supplemental tool for instructors, combining algorithms used in machine learning with adaptive methods used to track facial changes during a longer period of time.

On the other hand, in [4], the proposal uses fingerprint verification technique. They propose a system in which fingerprint verification is done by using extraction of minutiae technique and the system that automates the whole process of taking attendance.

Since biometrics are concerned with the measurements of unique human physiological or behavioral characteristics, the technology has been used to verify the identity of users. It is becoming critical to be able to monitor the presence of the authenticated user throughout a session. Thus, another proposal [5], discusses a prototype system that uses facial recognition technology to monitor authenticated user or students. A neural network-based algorithm was implemented to carry out face detection, and an eigenface method was employed to perform facial recognition. The experimental results demonstrate the feasibility of near-real-time continuous user verification for high-level security information systems. [5]

We noticed that most proposals do involve applications being used by the instructor during class. Hence, if the attendance system requires some action from the instructor, then the class time will be disturbed each time the instructor allows some late students into the class. On the other hand, our proposal does require the instructor to do nothing extra beyond presenting the slides of the course to the students. Hence, students may register their presence at any time they wish during the class, while having in mind that registration times are recorded. 


\section{QR CODE: Quick RESPONSE CODE}

QR code (abbreviated from Quick Response Code) is the trademark for a type of matrix barcode (or two-dimensional bar code) first designed for the automotive industry in Japan. Bar codes are optical machine-readable labels attached to items that record information related to the item. It was initially patented; however, its patent holder has chosen not to exercise those rights. Recently, the QR Code system has become popular outside the automotive industry due to its fast readability and greater storage capacity compared to standard UPC barcodes. The code consists of black modules (square dots) arranged in a square grid on a white background. The information encoded may be made up of four standardized types ("modes") of data (numeric, alphanumeric, byte / binary, Kanji) or, through supported extensions, virtually any type of data

A QR code, as shown in Fig.1 is read by an imaging device, such as a camera, and formatted algorithmically by underlying software using Reed-Solomon error correction until the image can be appropriately interpreted. Data is then extracted from patterns present in both horizontal and vertical components of the image. The QR features are listed in table 1. Figure shows a sample of an unencrypted $\mathrm{QR}$ code that will be needed by the proposed system.

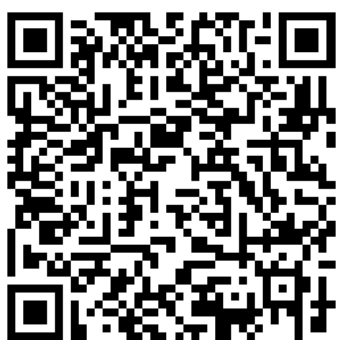

Fig. 1. Quick Response Code

\section{THE PROPOSED SYSTEM}

The system lies between online learning and traditional learning as a facilitation for the attendance record-keeping process, in a way that enriches the lecture time so that it can better be utilized in giving useful materials rather than wasting the time taking attendance.

The system requires a simple login process by the class instructor through its Server Module to generate an encrypted $\mathrm{QR}$ code with specific information. This can be done at any time before the class. During the class, or at its beginning, the instructor displays an encrypted $\mathrm{QR}$ code to the students. The students can then scan the displayed QR code using the system Mobile Module, provided to them through the smartphone market by the university. Along with the student's facial image captured by the mobile application at the time of the scan, the Mobile Module will then communicate the information collected to the Server Module to confirm attendance. The whole process should take less than a minute for any student as well as for the whole class to complete their attendance confirmation. Smartphones may communicate with the server via either the local $\mathrm{Wi}-\mathrm{Fi}$ coverage offered by the institution or through the internet.
TABLE I. CAPACITY, FEATURES, AND STANDARDS FOR QR CODE

\begin{tabular}{|l|l|}
\cline { 2 - 2 } \multicolumn{1}{c|}{} & QR Code \\
\hline $\begin{array}{l}\text { Developer } \\
\text { (country) }\end{array}$ & $\begin{array}{l}\text { DENSO } \\
\text { (Japan) }\end{array}$ \\
\hline Numeric & 7,089 \\
\hline Alphanumeric & 4,296 \\
\hline Binary & 2,953 \\
\hline Kanji & 1,817 \\
\hline Major Features & $\begin{array}{l}\text { Large } \\
\text { capacity } \\
\text { Small } \\
\text { printout size } \\
\text { High speed } \\
\text { scan }\end{array}$ \\
\hline Standards & $\begin{array}{l}\text { AIM } \\
\text { International } \\
\text { JIS } \\
\text { ISO }\end{array}$ \\
\hline
\end{tabular}

As mentioned earlier, the system is composed of two modules: the Server and the Mobile Modules. The Server Module can be integrated with the eLearning platform used by the institution or it can be a separate application depending on the choice of the developer. The following subsection will describe the tasks for each module.

\section{A. Server Module}

The Server Module performs the following tasks:

- Mediates students' attendance requests with the eLearning system.

- Generates a QR code for the instructor

- Runs Identity check

- Runs Location check

An example of an eLearning platform, which is an open source application that has become very popular in recent years, is Moodle. Moodle is used by many institutions worldwide. Among its features is Taking Attendance. It allows the instructors to take attendance online by calling names and checking online the appropriate checkbox next the student name. The checkboxes or radio-buttons offered are marked by $\mathrm{P}$ for Present, L for Late, and A for Absent. One of the Server Module jobs is to automatically mark the right radio-button on the attendance sheet list.

This module can be developed as a plug-in module to Moodle. When a student sends his/her information via the Mobile Module to the server, as shown in Figure 3, the server in turn sends the Student ID, the lecture date and time, the attendance status, and a small size image of the student face captured by the Mobile Module to the eLearning platform. This way, the Moodle plug-in will save the transaction as well as register the appropriate attendance status. To generate the QR code, the instructor logs in the Server Module or the eLearning system (if the Server Module is developed as part of the eLearning system), to enter the information needed by the system into the QR code. 


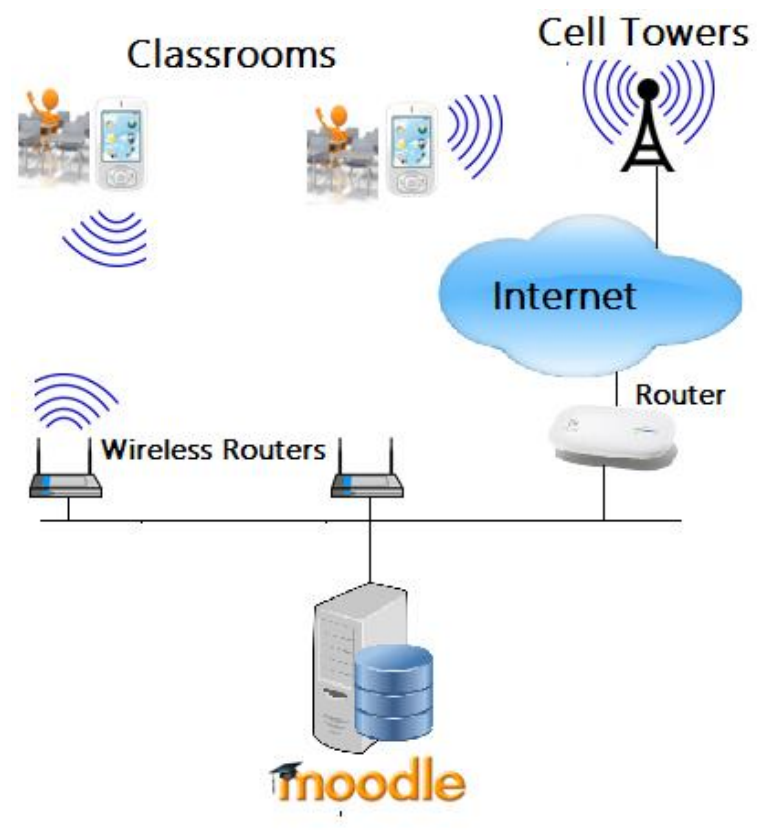

Fig. 2. The proposed system infrastructure

The instructor may choose then to encrypt this code depending on the level of protection needed. The QR code, with or without encryption, will include the following information:

- Course and section ID

- Date and beginning time of the lecture

- Instructor name

- Some random passcode

The information in Fig.1 can be interpreted as shown in Table 2:

TABLE II. TEXT EXTRACTED From A QR CODE

\begin{tabular}{|c|}
\hline Course: 1301120 \\
Section: 1 \\
Instructor: Prof. Nael Hirzallah \\
Date: $1 / 1 / 2014$ \\
BeginTime: 10:00AM \\
Passcode: $6 F g T 4 E$
\end{tabular}

The instructors in turn copy this QR code and paste it on the first slide to be displayed in the lecture. If the instructor policy is to allow late students in his class and would like to mark them as present or late, then the QR code should also be copied on one of the four corners of as many slides as the instructor wishes.

When the students are in class, the first thing that should be done is to pull out their smartphones, open the Mobile Module, and scans the QR code. Figure 3 shows the QR code in one of the slides of the lecture.

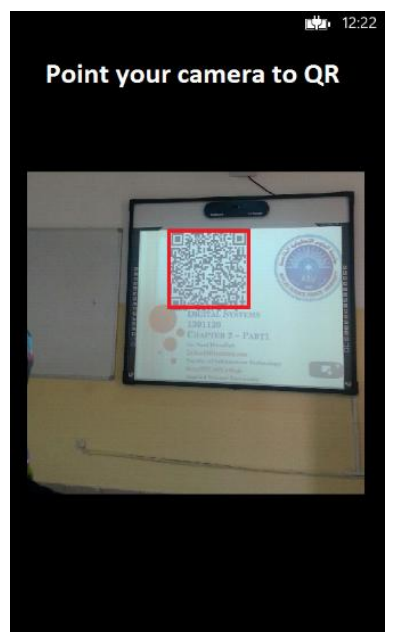

Fig. 3. Snapshot of the first slide

The third task of the Server Module is to run an identity check on the registered students. This is done by comparing the facial image sent per transaction and that stored image on file for the student in question. A matching score will be added to the attendance sheet so the instructor could perform a manual check either during the lecture or after the lecture. The identity check, or image comparisons, can be done once the attendance registration transaction is received, or at a later scheduled time. Although it is recommended to perform the job once the student signs in with the system to be marked as present, but if the number of students and concurrent lectures are large compared to the speed of the server, then the job could be performed say at a random instant in the second half of the lecture. The purpose of this job, is to allow the instructor to check the results of the identity check before the end of the lecture, if he/she wishes to do so.

Finally, a location check will be performed. This task will be discussed later.

\section{B. Mobile Module}

The Mobile Module is the part that students usually install on their smart phones. This could also be integrated with the Mobile part of the eLearning platform, or a standalone application that communicates with the Server Module. As mentioned earlier, the communication will be through the local Wi-Fi network, or it could be through the internet.

As depicted in Figure 4, once the student sees the QR code on the screen, he/she opens the Mobile application. If it is the first time after restarting the Smartphone, the system requests the student to enter a username and password. Once logged in, the system prompts the student to click on the start button. The system will then capture the face of the student. The facial image will be checked against standard facial conditions, such as locating the eyes. Once the image is accepted, the system requests the user to scan a $\mathrm{QR}$ code within a very short time. Once the code is scanned, the system sends the information to the server and resumes working in the background. With that, the process is considered completed. The server in turn will send back an acknowledgment that the process is complete. 


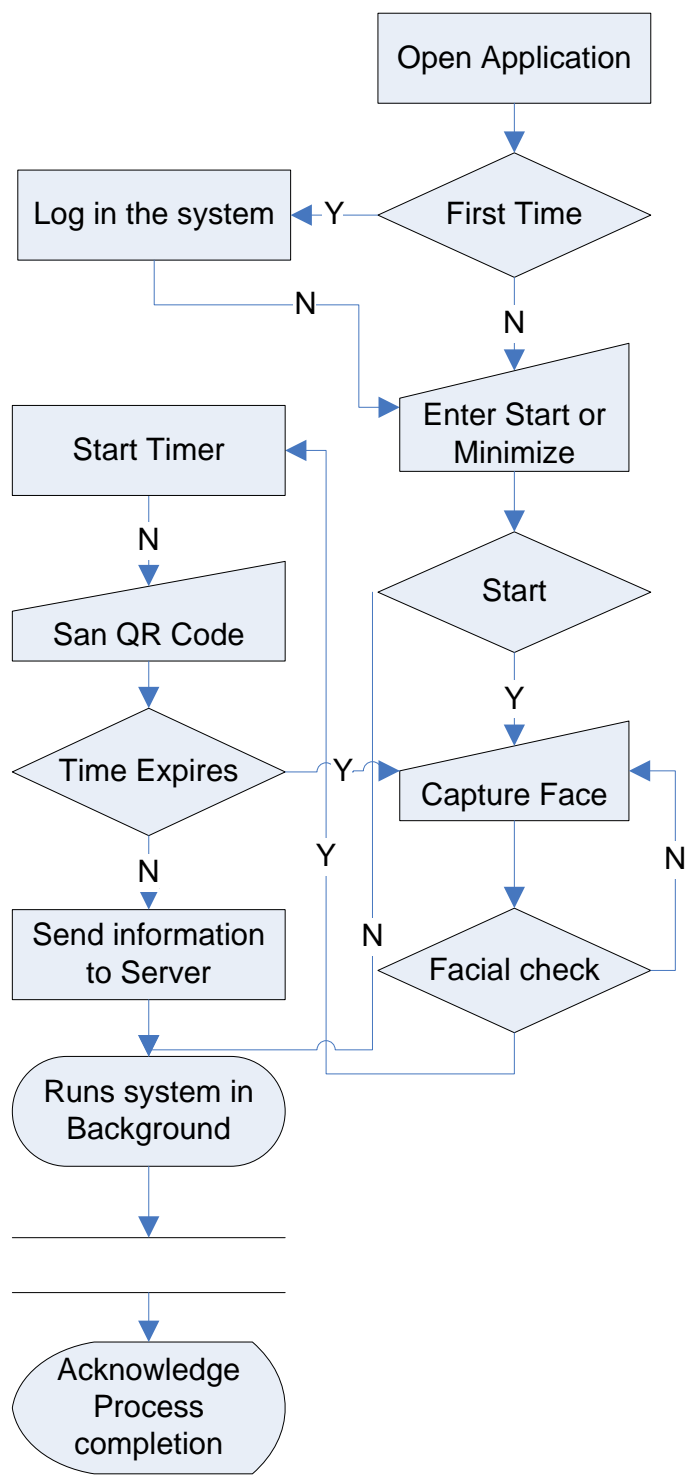

Fig. 4. Mobile Module Flow Chart

As stated in the previous section, the server verifies the identity of the students by running a facial match. The matching weight along with the facial image will be stored against the presence status of the student. The instructor may recheck any of the student's presence during the lecture by manually checking the updated attendance list that shows the matching weights during or after class.

\section{ANALYSIS}

Standard security procedures require a simple username and password. Such information is called "Something you know" or "What you know". Thus, it is easy for unauthorized users to gain access to a user's private data such as personal and financial details and then use that information to commit fraudulent acts.

Using a username and password together with a piece of hardware or device that only the user has makes it harder for potential intruders to gain access and steal that person's personal data or identity.
The proposed system will need three steps from each student. These steps are opening the application, capturing the face, and scanning the QR code. The system uses multi-factor authentication to authenticate students. These are "something you know" which is represented by username and password, "something you have" which is represented by the Smartphone owned by the student, and finally "something you are" which is represented by matching the facial image of the student. Thus, unauthorized users are not easy to get access to changing the presence status of one student.

However, for taking attendance, the challenge is in the fact that the system must guarantee that such a process do really take place within the classroom and not outside. The only fraudulent act that may happen is when a student sitting outside a classroom receives an image of the displayed QR code from a student sitting inside, via some communication medium such as email. This may be enough to simulate the process as if it taken place inside the classroom. To prevent this, the location of the Mobile phone information will accompany the information sent to the server.

Currently, Global Navigation Satellite Systems, (GNSS) receivers are becoming more and more sensitive due to ceaseless progress in chip technology and processing power. High Sensitivity GNSS receivers are able to receive satellite signals in most indoor environments and attempts to determine the 3D position indoors have been successful, [6].

Besides increasing the sensitivity of the receivers, the technique of A-GPS can be used, where the almanac and other information are transferred through a mobile phone. Furthermore, as smart phones embrace always-on, ubiquitous location, location-based sensor fusion will become a standard feature.

ABI Research's report, "Location-based Sensor Fusion: Companies, Technologies, and Revenue Opportunities," [7] outlines how sensor fusion will evolve to support indoor location and the companies best placed to succeed in this space.

Once the location information is sent to the server, the center of class will be calculated for all the smart phones locations received until the time of the check. The distance from the center of each Smartphone will be recorded along with the facial matching weights. This will allow the instructor to do a check on the awkward positions of the phones or awkward matching weights during class. In other words, the following information will be communicated to the server per transaction:

- Student ID from application account

- Class and time details from QR code

- Smartphone location from device

The list of attendance that shows the status of the student versus the Facial matching weight and Distance can be presented to the instructor upon his/her request. Figure 5 shows an updated attendance list after both facial image matching and distance calculations processes are performed. 


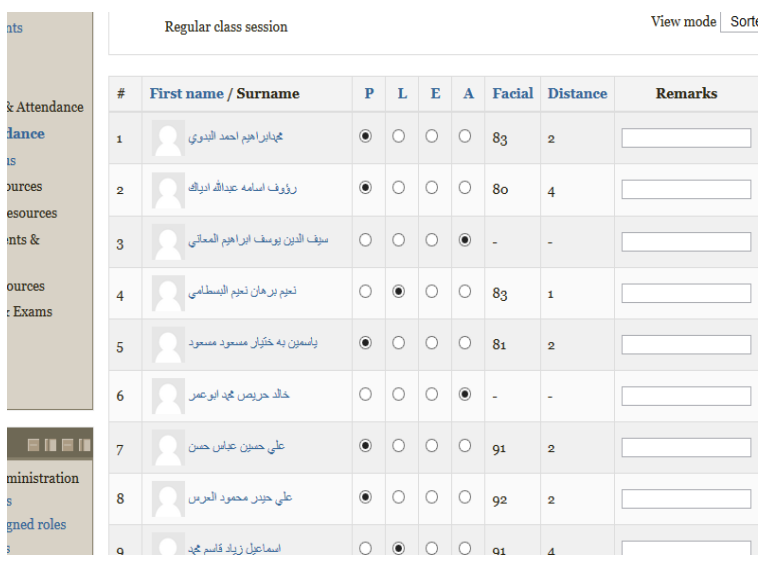

Fig. 5. Updated Attendance Sheet

Figure 6 shows snapshots of the Mobile application.

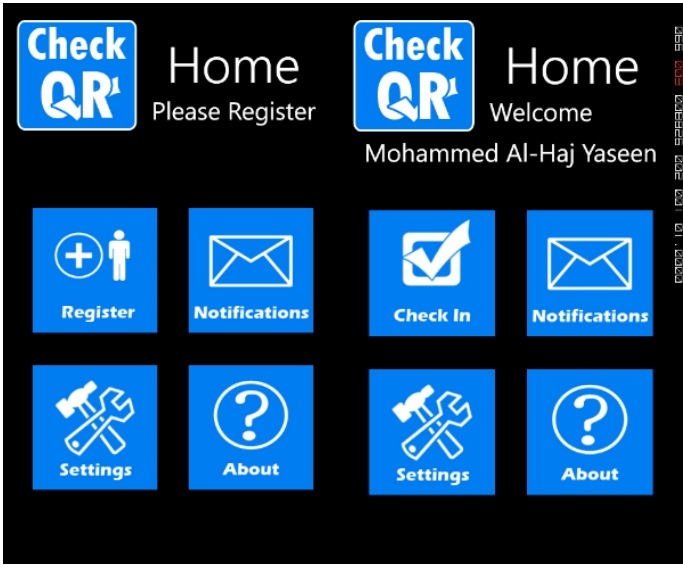

Fig. 6. Snapshots of the Mobile Module

\section{CONCLUSION}

These days it is required to keep up with the latest technologies, especially in the field of education. Educational institutions have been looking for ways to enhance the educational process using the latest technologies. Looking at the existing situation, we have thought of using the mobile technology to efficiently benefit from the complete assigned time assigned to a lecture. Time taken by instructors to take attendance may be viewed sometimes as a waste of the lecture time, especially when classes are big. For that, we have proposed a way to automate this process using the students' devices rather than the instructor's device. In other words, the instructor need not do anything extra during the class beyond presenting the slides of the subject to be taught to the students.

The proposed system allows fraud detection based on the GPS locations as well as the facial images taken for each student.

\section{ACKNOWLEDGMENT}

The authors wish to acknowledge the financial support received from Applied Science University that helped in publishing this article.

\section{REFERENCES}

[1] Smartphone Users Around the World - Statistics and Facts, http://www.go-gulf.com/blog/smartphone/ visited on February 12, 2014

[2] Jamil, T. ; Dept. of Electr. \& Comput. Eng., Sultan Qaboos Univ., Al Khod, Oman, Automatic attendance recording system using mobile telephone, Telecommunications Forum (TELFOR), 2011 19th 1297 1299

[3] Shehu, V. ; Contemporary Sci. \& Technol., South East Eur. Univ., Tetovo, Macedonia ; Dika, A. , Using real time computer vision algorithms in automatic attendance management systems, Information Technology Interfaces (ITI), 2010 32nd International Conference on 397 $-402$

[4] Saraswat, Chitresh; Kumar, Amit, An Efficient Automatic Attendance System using Fingerprint Verification Technique. International Journal on Computer Science \& Engineering . 2010, Vol. 2 Issue 2, p264-269

[5] Qinghan Xiao ; Interdept. Biometrics Working Group, Gov. of Canada, ON ; Xue Dong Yang, A facial presence monitoring system for information security, Computational Intelligence in Biometrics: Theory, Algorithms, and Applications, 2009. CIB 2009. IEEE Workshop on, March 30 2009-April 2 2009, 69 - 76

[6] Indoo Positioning: http://en.wikipedia.org/wiki/Indoor_positioning_system\#cite_note-15 visited on January 10, 2014

[7] Location-based Sensor Fusionhttps://www.abiresearch.com/research/product/1015686-locationbased-sensor-fusion-companies-tec/visited on January 10,2 